\title{
On the Time-Like and Space-Like Components of Majorana Field
}

\author{
Luca Nanni \\ University of Ferrara, 44100-Ferrara, Italy \\ Email: luca.nanni68@gmail.com
}

\begin{abstract}
The infinite-component Majorana field is a promising tool for investigating phenomena that cannot yet be explained within the Standard Model framework, such as the oscillation of neutrino flavours, the origin of particle mass and the physical nature of dark matter. In this study, we demonstrated that the Lagrangian density of the fermionic Majorana field can be represented as the sum between the Dirac field with positive energy and the infinite Dirac-like tachyonic fields with negative frequency. Particularly, we demonstrated that the tachyonic components of the field are obtained by the superluminal Lorentz transformation (SLT) of the bradyonic components. Using this result, we obtained the explicit form of the SLT matrices, tachyonic creation and annihilation operators of the four-spinors on which they act. Thus, the Majorana field becomes the sum of the infinite Dirac fields transformed by the finite SLT matrices, which are a familiar tool in the physics of half-integer spin particles. A decay mechanism for an ordinary particle with tachyonic pair production is also proposed. The approach used in this study is an attempt to investigate particle physics beyond the Standard Model.
\end{abstract}

Keywords: Infinite-dimensional matrices; Dirac field; superluminal Lorentz transformations; tachyon; tachyonic pair production.

\section{Introduction}

In 1932, Majorana formulated an equation for a particle with an arbitrary spin [1] that is invariant with respect to the infinitesimal transformations of the Lorentz group [2-3], and whose (time-like) solutions only have positive energy. Unlike Dirac, Majorana does not require that the equation considers the energy conservation constraint. With this contribution, Majorana advanced the field of quantum physics far more than the preceding scientific research over approximately two decades4. In fact, in its simple and elegant formalism, the Majorana equation includes the first infinite-dimensional representation of the Lorentz group (a result to which Weyl arrived only in 1937 [5]), the first discrete mass spectrum of particles, and the first theoretical prediction of tachyon particles with positive and negative frequency, which quantum physics had not considered at that time. However, in 1932, the positron was discovered by Anderson [6] (explained by the negative energy solutions of the Dirac [7] equation), while the neutron was discovered by Chadwick [8]. Hence, these discoveries eventually led to the formulation of the first theories of beta decay [9-10], which contributed toward undermining the correctness of the Majorana equation in favour of Dirac's equation. Therefore, the Majorana equation was relegated to oblivion on the year of its publication [11]. Moreover, the time-like solutions of the Majorana equation exhibit discrete mass spectra, which decrease as the spin increases. This theoretical prediction was not (and still has not been) confirmed experimentally in the 1930s [12].

The Majorana equation was reconsidered only years later, and particularly by Fradkin13 within the framework of hadronic physics [14-15]. However, any attempt to incorporate the Majorana equation into a field theory has always led to conflicting results that have not been in line with current quantum theories. For instance, Grodsky demonstrated that the quantization of the Majorana field led to the violation of the CTP invariance and spin statistic theorem16, while Surdashan demonstrated that, by using an appropriate approach, the quantization of the Majorana field becomes compliant with the spin statistic theorem again, and that the space-like solutions can be treated similarly to the time-like solutions [17]. 
In a recent study [18], it was demonstrated that the Majorana field may be reinterpreted as a linear combination of a Dirac field with positive energy and infinite Dirac-like tachyon fields with negative frequencies (antitachyons) in the form of states where the half-integer spin has parallel $\mathrm{J}_{\mathrm{z}}$ components. This reinterpretation was based on the Majorana theorem19, according to which the dynamic of a system with an arbitrary spin can always be reduced to that of a composite system with a half-integer spin. By following this approach it is possible to quantize the entire field without violating the CTP invariance and the spin statistic theorem. Moreover, the Hamiltonian density is lower bounded, owing to the negative frequencies given by the tachyonic components.

The objective of this study was to demonstrate that the tachyonic components with negative energy are nothing more than Dirac fields transformed by the superluminal Lorentz transformation (SLT) matrices [20-22]. Therefore, the infinite-component Majorana field, constructed by infinite matrices, can be represented as an infinite sum of transformed Dirac fields, which is characterized by four-matrices and four-component spinors. In this paper, we present the explicit form of the SLT matrices, creation and annihilation operators, and spinors on which they act. Within this framework, the Majorana field is an attempt to investigate particle physics beyond the Standard Model by replacing the restricted theory of relativity with a theory extended to superluminal motions [23-27]. This new Model could facilitate the formulation of new theories that are capable of answering unsolved questions, such as the origin of the particle mass spectra or the flavour oscillations of the neutrino [28-32].

\section{Preliminaries}

The constraint imposed by Majorana to formulate a quantum theory for particles with arbitrary spin, i.e., an equation whose solutions have only positive energy, leads to a discrete mass spectrum that decreases as the value of the intrinsic angular momentum increases [1], as follows:

$$
m_{J}=\frac{m_{0}}{(J+1 / 2)}
$$

where $\mathrm{m}_{0}$ is the rest mass of the particle and $J$ is the particle spin. In this study, we were interested only in neutral fermions; therefore, $J$ is a half-integer value and an electrodynamic interaction does not occur. Using the energy-momentum relationship, the energy difference between a state with spin $J$ and the fundamental state with $J_{0}=1 / 2$ can be expressed as follows:

$$
E_{J}^{2}-E_{J_{0}}^{2}=\left[p_{J}^{2}-p_{J_{0}}^{2}\right] c^{2}-\frac{(J+1 / 2)^{2}-1}{(J+1 / 2)^{2}} m_{0}^{2} c^{4}
$$

If the reference frame is that of the particle in the fundamental state, then Eq. 2 becomes as follows:

$$
\Delta E_{\left(J-J_{0}\right)}^{2}=p_{J}^{2} c^{2}-\frac{(J+1 / 2)^{2}-1}{(J+1 / 2)^{2}} m_{0}^{2} c^{4}
$$

Eq. 3 is the peculiar energy-momentum relationship of a tachyon with imaginary mass given by:

$$
\mu_{J}=i \frac{\sqrt{(J+1 / 2)^{2}-1}}{(J+1 / 2)} m_{0}
$$

Eq. 3 suggests that the solutions of the Majorana equation with spin values greater than $1 / 2$ describe the excited states of the particle, where the fermion in the fundamental state interacts with a tachyon in a multiplet state $\left[2\left(J-J_{0}\right)+1\right]$ where $J_{z}=\left|2\left(J-J_{0}\right)+1\right|$. Therefore, the Majorana excited state is a composite particle. This idea has already been considered by other authors [33]. In the formalism of the quantum field theory, we may consider the Majorana excited states with spin J as an interaction between a bradyonic field with spin $J_{0}$ and a tachyon field with $J_{z}=\left|2\left(J-J_{0}\right)+1\right|$.

The occupation probability of an excited state $\mathrm{J}$ is expressed as follows [34]:

$$
P_{J}=\sqrt{\beta^{n}-\beta^{(n+1)}} \quad \text { where } n=(J+1 / 2)
$$

where $\beta$ is the relativistic factor. The energy at which the interaction between the two fields becomes maximum can be determined by setting $\partial P_{J} / \partial \beta=0$ to obtain the following equation:

$$
\beta_{J}^{(\max )}=\frac{(J-1 / 2)}{(J+1 / 2)}
$$


Considering that the particle is at rest in the fundamental state (and thus $\gamma_{J_{0}}=1$ ), the energy of the tachyon corresponding to the maximum interaction is expressed as follows:

$$
\varepsilon_{J}^{2}=\gamma_{t}^{2} \mu_{J}^{2} c^{4}=E_{J}^{2}-E_{J_{0}}^{2}=\gamma_{J}^{2} m_{0}^{2} c^{4}-\gamma_{J_{0}}^{2} m_{0}^{2} c^{4}
$$

where $\gamma_{t}^{2}$ is the tachyonic Lorentz [35] factor and $\gamma_{J}^{2}$ must be calculated using Eq. 6. From Eq. 7 we obtain the explicit form of $\gamma_{t}^{2}$, as follows:

$$
\gamma_{t}^{2}=\left(1-u_{t}^{2} / c^{2}\right)^{-1 / 2}=\frac{(J+1 / 2)^{2}\left[(J+1 / 2)^{2}-2\right]}{2\left[(J+1 / 2)^{2}-1\right]}
$$

This relationship gives us the tachyon velocity corresponding to the energy of the maximum interaction, as follows:

$$
u_{t}^{2}=c^{2}\left[1+\frac{2\left[(J+1 / 2)^{2}-1\right]}{(J+1 / 2)^{2}\left[(J+1 / 2)^{2}-2\right]}\right]=c^{2} \chi_{J}^{2}
$$

As shown in Fig. 1, the numerical coefficient $\chi_{J}$, which represents the relativistic factor of the tachyonic regime, rapidly tends to one as the spin increases.

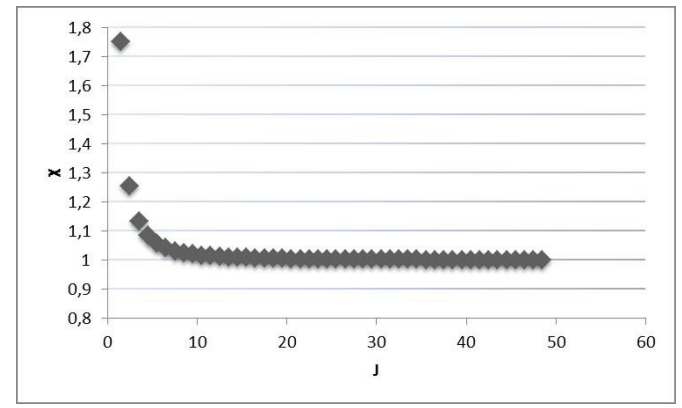

Figure 1. Numerical factor $\chi_{J}$ vs. particle spin $J$.

This means that the probability of the interaction between the bradyon and the tachyon does not have negligible values only when both particles have very high energy, that is, in an ultrarelativistic regime.

\section{Majorana Lagrangian Field}

As proposed in a recent paper [18], the Lagrangian density of the Majorana field may be expressed as the sum between the Dirac field with positive energy and the infinite Dirac-like tachyonic fields with negative frequencies [35], as follows:

$$
\mathcal{L}_{J}=\bar{\psi}_{D_{+}}\left(i \gamma^{\mu} \partial_{\mu}-m_{0}\right) \psi_{D_{+}}+\bar{\psi}_{t_{-}}\left(i \gamma^{\mu} \partial_{\mu}+i \frac{\sqrt{(J+1 / 2)^{2}-1}}{(J+1 / 2)} m_{0}\right) \psi_{t_{-}}
$$

The Dirac field operators are expressed as follows:

$$
\left\{\begin{array}{c}
\bar{\psi}_{D_{+}}=\int \frac{d^{3} p}{(2 \pi)^{3}} \frac{1}{\sqrt{2 E}} \sum_{s}\left(a_{p}^{s}\right)^{\dagger} \bar{u}_{p}^{S} \exp \{-i(k x-\omega t)\} \\
\psi_{D_{+}}=\int \frac{d^{3} p}{(2 \pi)^{3}} \frac{1}{\sqrt{2 E}} \sum_{s} a_{p}^{s} u_{p}^{S} \exp \{i(k x-\omega t)\}
\end{array}\right.
$$

where the superscript $s$ represents the two possible spin z-components $J_{z}= \pm 1 / 2$. The creator and annihilator operators must satisfy the anticommutation relationship, as follows:

$$
\left\{a_{p}^{r},\left(a_{q}^{s}\right)^{\dagger}\right\}=(2 \pi)^{3} \delta^{3}(p-q) \delta_{r s}
$$

Because only positive energy solutions are considered, $\gamma^{0}$ represents the unit matrix. The Dirac-like tachyonic operator fields with negative frequencies are expressed as follows: 


$$
\left\{\begin{array}{c}
\bar{\psi}_{t_{-}}=\int \frac{d^{3} p}{(2 \pi)^{3}} \frac{1}{\sqrt{2 \varepsilon}} \sum_{s}\left(t_{p}^{s, J}\right)^{\dagger} \bar{w}_{p}^{S} \exp \left\{-i\left(k_{t} x-\omega_{t} t\right)\right\} \\
\psi_{t_{-}}=\int \frac{d^{3} p}{(2 \pi)^{3}} \frac{1}{\sqrt{2 \varepsilon}} \sum_{s} t_{p}^{S, J} w_{p}^{S} \exp \left\{i\left(k_{t} x-\omega_{t} t\right)\right\}
\end{array}\right.
$$

where the superscript $s$ always represents the two possible spin components $J_{z}= \pm 1 / 2$. Obviously, operators $\left(t_{p}^{s}\right)^{\dagger}$ and $t_{p}^{s}$ must comply with the anticommutation relationship expressed by Eq. 12. Let us reiterate that the spin z-components of tachyons must be parallel to the spin z-component of the bradyon. Therefore, to construct particles with an increasing spin, it is necessary for operator $\left(a_{p}^{s}\right)^{\dagger}$ to create a number of $(2 J-1)$ tachyons with negative frequency such that their total mass is $\mu_{J}$. For instance, the state with $J=3 / 2$ is given by the interaction between the spin-1/2 bradyon and the ensemble of the two spin- $1 / 2$ antitachyons with a total mass of $\mu_{3 / 2}=\mathrm{i} \frac{\sqrt{3}}{2} \mathrm{~m}_{0}$. In accordance with this mechanism, we can write the tachyonic spinors as follows:

$$
\left\{\begin{aligned}
\bar{w}_{p}^{S, J} \exp \left\{-i\left(k_{t} x-\omega_{t} t\right)\right\} & =\prod_{\rho=1}^{2 J-1} \bar{w}_{p_{\rho}}^{1 / 2} \exp \left\{-i\left(k_{t}^{\rho} x-\omega_{t}^{\rho} t\right)\right\} \\
w_{p}^{s, J} \exp \left\{i\left(k_{t} x-\omega_{t} t\right)\right\} & =\prod_{\rho=1}^{2 J-1} w_{p_{\rho}}^{1 / 2} \exp \left\{i\left(k_{t}^{\rho} x-\omega_{t}^{\rho} t\right)\right\}
\end{aligned}\right.
$$

Because the reference frame is that of the center of mass, the bradyonic and tachyonic wave numbers and frequencies are expressed as follows:

$$
\left\{\begin{aligned}
k=K_{J_{0}}=0 \quad ; \quad \omega=\omega_{J_{0}}=\frac{m_{0} c^{2}}{\hbar} \\
k_{t}=\frac{1}{\hbar} \sqrt{\left(p_{J}^{2}-p_{J_{0}}^{2}\right)} c=\frac{1}{\hbar} p_{J} c \quad ; \quad \omega_{t}=\frac{1}{\hbar} \sqrt{p_{J}^{2} c^{2}-\frac{(J+1 / 2)^{2}-1}{(J+1 / 2)^{2}} \omega_{J_{0}}^{2}}
\end{aligned}\right.
$$

where the following inequality must hold:

$$
p_{J}^{2} c^{2}>\frac{(J+1 / 2)^{2}-1}{(J+1 / 2)^{2}} \omega_{J_{0}}^{2}
$$

Eqs. 14 and 15 indicate that the exponential functions $\exp \{ \pm i(k x-\omega t)\}$ and $\exp \left\{ \pm i\left(k_{t} x-\omega_{t} t\right)\right\}$ are connected by the following functional relationship:

$$
\exp \left\{-i\left(k_{t} x-\omega_{t} t\right)\right\}=g\left(\varepsilon_{J}\right) \exp \{-i(k x-\omega t)\}=g\left(\varepsilon_{J}\right) \exp \left\{\mp i \omega_{J_{0}} t\right\}
$$

where:

$$
\varepsilon_{J}=\frac{\sqrt{(J+1 / 2)^{2}-1}}{(J+1 / 2)}
$$

whose value ranges between zero and one. To find the function $g\left(\varepsilon_{J}\right)$, let us consider the following equations:

$$
\left\{\begin{array}{c}
k_{t}=\gamma_{t}\left|\mu_{J}\right| c=\left(\chi_{J}^{2}-1\right)^{-1 / 2} \frac{4 \sqrt{(J+2)}}{(J+1)(J+3)} \frac{m_{0} c}{\hbar}=\left(\chi_{J}^{2}-1\right)^{-1 / 2} \varphi_{J} k_{\text {Plank }} \\
\omega_{t}=\gamma_{t}\left|\mu_{J}\right| c^{2}=\left(\chi_{J}^{2}-1\right)^{-1 / 2} \varphi_{J} \omega_{\text {Plank }}
\end{array}\right.
$$

By substituting the explicit forms of $\mathrm{k}_{\mathrm{t}}$ and $\omega_{\mathrm{t}}$ in Eq. 17, we can easily obtain function $g\left(\varepsilon_{J}\right)$ as follows:

$$
g\left(\varepsilon_{J}\right)=\exp \left\{ \pm i\left(\chi_{J}^{2}-1\right)^{-1 / 2} \varphi_{J}\left[k_{\text {Plank }} x-\frac{\left(\chi_{J}^{2}-1\right)^{-1 / 2} \varphi_{J} \mp 1}{\left(\chi_{J}^{2}-1\right)^{-1 / 2} \varphi_{J}} \omega_{\text {Plank }} t\right]\right\}
$$

Therefore, the Lagrangian expressed by Eq. (10) represents the ensemble of the infinite subluminal particles with decreasing mass, which are formed by the one time-like component and the $(2 J-1)$ space-like components, all with a parallel spin-of $1 / 2$.

The theory that we are formulating must be invariant under a SLT. In this context, the tachyonic operators and spinors can be seen as the result of the appropriate SLT transformations of time-like operators and spinors. This topic will be discussed in detail in the next section. 


\section{Superluminal Lorentz Transformation between Time-like and Space-like Components}

In this section, we will use the formalism proposed by Recami for the SLTs [21, 22, 27]. The transformation matrices are antiorthogonals, as follows:

$$
\Lambda_{J}^{T} \Lambda_{J}=-\mathbb{1}
$$

Moreover, it can be easily proven that the inverse matrix is equal to the transpose matrix with the opposite sign, as follows:

$$
\Lambda_{J}^{T} \Lambda_{J} \Lambda_{J}^{-1}=-\mathbb{1} \Lambda_{J}^{-1} \quad \text { and } \quad \Lambda_{J}^{T} \Lambda_{J} \Lambda_{J}^{-1}=\Lambda_{J}^{T} \mathbb{1} \quad \Rightarrow \quad \Lambda_{J}^{-1}=-\Lambda_{J}^{T}
$$

Because the Lagrangian expressed by Eq. (10) must be invariant for the SLTs, the tachyonic creation and annihilation operators must be derived from the bradyonic ones through similarity transformations, as follows:

$$
\left(\Lambda_{J}\right)^{-1} a_{p}^{s} \Lambda_{J}=t_{p}^{s, j} \quad ; \quad\left(\Lambda_{J}\right)^{-1}\left(a_{p}^{s}\right)^{\dagger} \Lambda_{J}=\left(t_{p}^{s, J}\right)^{\dagger}
$$

The same consideration must be made for the tachyonic spinors, as follows:

$$
\left(\Lambda_{J}\right)^{-1} u_{p}^{s} \Lambda_{J}=w_{p}^{S, J} \quad ; \quad\left(\Lambda_{J}\right)^{-1} \bar{u}_{p}^{s} \Lambda_{J}=\bar{w}_{p}^{S, J}
$$

Using Eqs. (20) and (21), and property (18) we obtain the following relationships:

$$
\left\{\begin{array}{c}
t_{p}^{s, j} w_{p}^{s, J}=\left(\Lambda_{J}\right)^{-1} a_{p}^{s} \Lambda_{J}\left(\Lambda_{J}\right)^{-1} u_{p}^{s} \Lambda_{J}=-\Lambda_{J}^{T} a_{p}^{s} u_{p}^{s} \Lambda_{J} \\
\left(t_{p}^{s}\right)^{\dagger} \bar{w}_{p}^{s, J}=\left(\Lambda_{J}\right)^{-1}\left(a_{p}^{s}\right)^{\dagger} \Lambda_{J}\left(\Lambda_{J}\right)^{-1} \bar{u}_{p}^{s} \Lambda_{J}=-\Lambda_{J}^{T}\left(a_{p}^{s}\right)^{\dagger} \bar{u}_{p}^{s} \Lambda_{J}
\end{array}\right.
$$

Therefore, the tachyonic field with negative frequencies can be rewritten as follows:

$$
\left\{\begin{array}{l}
\bar{\psi}_{t_{-}}=-\Lambda_{J}^{T} \bar{\psi}_{D_{+}} \Lambda_{J} \\
\psi_{t_{-}}=-\Lambda_{J}^{T} \psi_{D_{+}} \Lambda_{J}
\end{array}\right.
$$

By substituting the transformed tachyonic fields in Eq. (10), we can write the Majorana Lagrangian as the infinite sum of the finite-dimensional Dirac-like fields as follows:

$$
\mathcal{L}_{M}=\bar{\psi}_{D_{+}}\left(i \gamma^{\mu} \partial_{\mu}-m_{0}\right) \psi_{D_{+}}+\sum_{J=3 / 2}^{\infty} \Lambda_{J}^{T} \bar{\psi}_{D_{+}}\left(i \Lambda_{J} \gamma^{\mu} \Lambda_{J}^{T} \partial_{\mu}-i \varepsilon_{J} m_{0}\right) \psi_{D_{+}} \Lambda_{J}
$$

where only the well-known four-Dirac-spinor and the four-Dirac-gamma-matrices are used.

The transformations expressed by Eq. (20), in combination with the property expressed by Eq. (18), are useful in proving that the tachyonic creator and annihilator operators satisfy the anticommutation relationship, as follows:

$$
\left\{t_{p}^{r, J},\left(t_{q}^{s, J}\right)^{\dagger}\right\}=\Lambda_{J}^{T} a_{p}^{s} \Lambda_{J} \Lambda_{J}^{T}\left(a_{p}^{s}\right)^{\dagger} \Lambda_{J}-\Lambda_{J}^{T}\left(a_{p}^{s}\right)^{\dagger} \Lambda_{J} \Lambda_{J}^{T} a_{p}^{s} \Lambda_{J}=-\left\{a_{p}^{r},\left(a_{q}^{s}\right)^{\dagger}\right\}
$$

The SLTs preserve the Clifford algebra of the gamma-Dirac-matrices as follows:

$$
\left\{\begin{array}{c}
\left\{\Lambda_{J} \gamma^{\mu} \Lambda_{J}^{T}, \Lambda_{J} \gamma^{v} \Lambda_{J}^{T}\right\}=-2 g^{\mu v} \mathbb{1} \\
\Lambda_{J} \gamma^{0} \Lambda_{J}^{T}=\Lambda_{J}\left(\gamma^{0}\right)^{\dagger} \Lambda_{J}^{T} \text { and } \Lambda_{J} \gamma^{\mu} \Lambda_{J}^{T}=\Lambda_{J}\left(\gamma^{\mu}\right)^{\dagger} \Lambda_{J}^{T} \\
\Lambda_{J} \gamma^{0} \Lambda_{J}^{T} \Lambda_{J} \gamma^{0} \Lambda_{J}^{T}=-\mathbb{1} \text { and } \Lambda_{J} \gamma^{\mu} \Lambda_{J}^{T} \Lambda_{J} \gamma^{\mu} \Lambda_{J}^{T}=\mathbb{1}
\end{array}\right.
$$

Thus, this proves that the algebraic structure of the formulated theory is completely consistent with the physical-mathematical foundation of the fermionic fields theory described in the Standard Model.

\section{$5 \quad$ SLT Matrices of Majorana Field}

To complete our theory we need to find the explicit form of the $\Lambda_{\mathrm{J}}$ matrices. Recami proposes the following Hermitian form for the SLT matrices [22]: 


$$
\Lambda_{S L T}=\eta\left(\begin{array}{ccrc}
\gamma & i \gamma u_{x} / c & i \gamma u_{y} / c & i \gamma u_{z} / c \\
-i \gamma u_{x} / c & \delta+\alpha u_{x}^{2} / u^{2} & \alpha u_{x} u_{y} / u^{2} & \alpha u_{x} u_{z} / u^{2} \\
-i \gamma u_{y} / c & \alpha u_{x} u_{y} / u^{2} & \delta+\alpha u_{y}^{2} / u^{2} & \alpha u_{y} u_{z} / u^{2} \\
-i \gamma u_{z} / c & \alpha u_{x} u_{z} / u^{2} & \alpha u_{y} u_{z} / u^{2} & \delta+\alpha u_{z}^{2} / u^{2}
\end{array}\right)
$$

where $u_{x}, u_{y}$, and $u_{z}$ are the three components of the tachyonic velocity expressed by Eq. (9), while the following relationships hold:

$$
\left\{\begin{array}{cl}
\delta=\sqrt{\left(1-\operatorname{tg}^{2} \theta\right) /\left|1-\operatorname{tg}^{2} \theta\right|} ; & \gamma=\left|1-\operatorname{tg}^{2} \theta\right|^{-1 / 2} \quad ; \quad \operatorname{tg} \theta=\beta \\
\eta=\delta^{2} \frac{\cos \theta}{|\cos \theta|} \quad ; \quad \alpha=\gamma-\delta &
\end{array}\right.
$$

By using the explicit form of the relativistic factor $\chi_{J}$ for the tachyonic regime, the numerical parameters expressed by Eq. (28) become as follows:

$$
\left\{\begin{array}{c}
\delta=i \quad \gamma=i\left(\chi_{J}^{2}-1\right)^{-1 / 2} ; \quad \operatorname{tg} \theta=\chi_{J} \\
\eta=-1 ; \quad \alpha=i \frac{1-\sqrt{\left(\chi_{J}^{2}-1\right)}}{\sqrt{\left(\chi_{J}^{2}-1\right)}}=i \tau_{J}
\end{array}\right.
$$

Thus, the SLT matrix expressed by Eq. (27) takes the following form:

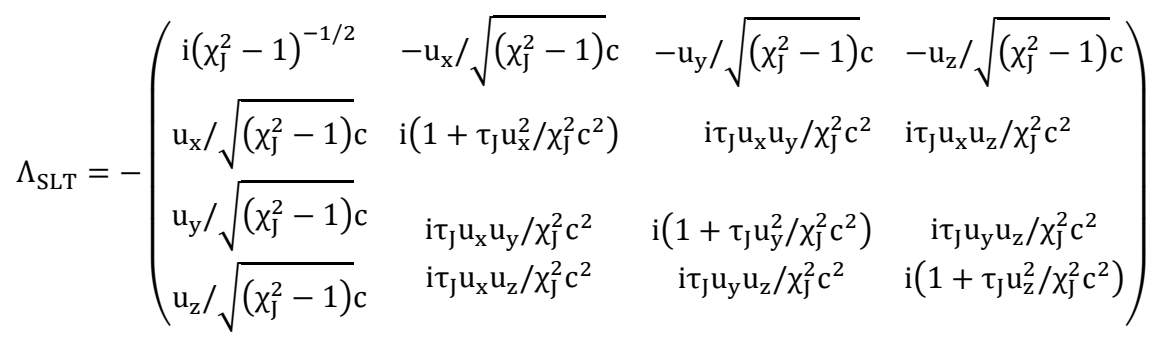

In the matrix expressed by Eq. (30), the tachyonic velocity components still remain unknown. To determine them, we must impose the constraint of $\Lambda_{J}^{T} \Lambda_{J}=-\mathbb{1}$, and consider that $u^{2}$ is equal to Eq. (9) to obtain the following relationships:

$$
\left\{\begin{array}{c}
u_{x} / c= \pm i \sqrt{\frac{\left(\chi_{J}^{2}-1\right) \tau_{J}^{2} u_{z}^{2}}{c^{2}}+\chi_{J}^{2}\left[\left(\chi_{J}^{2}-1\right)(2 \tau-1)\right]+\frac{\tau_{J}^{2} u_{z}^{2}}{c^{2}}} /\left(\chi_{J}^{2}-1\right) \tau_{J}^{2} \\
u_{x} / c \neq 0 \\
\forall-u_{z} / c \in \Re
\end{array}\right.
$$

Therefore, as expected, the tachyonic velocity $\mathrm{u}$ is quantized by Eq. (9). However, its components can take continuous values, provided that they comply with the relationships expressed in Eq. (31). Note that the components of tachyonic velocity may also be assumed to have negative and imaginary components [36].

\section{Discussion}

The maximum probability for the occupation of a given Majorana excited state takes significant values only when the particle velocity is close to that of light. In such an ultrarelativistic regime, quantum theory provides the formation of particle-antiparticle pairs [37] that are collinearly emitted with fourimpulses, as follows:

$$
p^{\mu}=\left(\frac{E}{c}, p\right)=\left(\frac{E_{1}}{c}, p_{1}\right)=\left(\frac{E_{2}}{c}, p_{2}\right) \text { such that } E=\sqrt{p^{2} c^{2}+m_{e}^{2} c^{4}}
$$

In the case of a neutral particle, such as a neutrino, the boson mediator of the process is $Z^{0}$ and its threshold momentum is expressed as follows:

$$
q^{2}=4 p^{\mu} p_{\mu}=4\left(p^{2} c^{2}+m_{e}^{2} c^{4}\right)-4 p^{2} c^{2}=4 m_{e}^{2} c^{4}
$$


Recently, Jentschura explained various data collected by the Ice Cube Collaboration and proposed a similar mechanism, where a superluminal neutrino decays into two collinear tachyonic pairs [38]. In the case where the two collinearly emitted tachyons have different energies, the following relationships hold:

$$
\left\{\begin{array}{c}
E_{1}=\sqrt{p_{1}^{2} c^{2}-m_{\mu}^{2} c^{4}} ; \quad E_{2}=\sqrt{p_{2}^{2} c^{2}-m_{\mu}^{2} c^{4}} \\
q^{2}=\left(\sqrt{p_{1}^{2} c^{2}-m_{\mu}^{2} c^{4}}+\sqrt{p_{2}^{2} c^{2}-m_{\mu}^{2} c^{4}}\right)^{2}-\left(p_{1} c+p_{2} c\right)^{2}=-4 m_{\mu}^{2} c^{4}
\end{array}\right.
$$

where $m_{\mu}$ is the tachyonic imaginary mass of the decaying neutrino. Because $q^{2}$ is negative, there is no lower threshold for tachyonic pair production. This result may be adapted to the proposed Majorana field theory; that is, in an ultrarelativistic regime a subluminal particle could decay into a tachyonantitachyon pair with parallel spin, whose total energy is always negative. Under this hypothesis, Eq. (34) becomes as follows:

$$
\left\{\begin{array}{c}
E_{1}=\sqrt{p_{1}^{2} c^{2}-\mu_{J}^{2} c^{4} / 2} ; \quad E_{2}=\sqrt{p_{2}^{2} c^{2}-\mu_{J}^{2} c^{4} / 2} \\
q^{2}=\left(\sqrt{p_{1}^{2} c^{2}-\mu_{J}^{2} c^{4} / 2}+\sqrt{p_{2}^{2} c^{2}-\mu_{J}^{2} c^{4} / 2}\right)^{2}-\left(p_{1} c+p_{2} c\right)^{2}=4 \frac{m_{0}^{2}}{(J+1 / 2)^{2}} c^{4}
\end{array}\right.
$$

Therefore, an ultrarelativistic bradyon in the fundamental state decays into a particle with lower mass and a tachyonic pair. In turn, the obtained particle may decay again into another lower mass particle with a new tachyonic pair production, and so on. This iterative mechanism fulfills all requirements discussed in Section 3. The probability that these decays will occur is expressed by Eq. 5 and declines progressively as the number of tachyonic pair production increases. Note that the term $q^{2}$ is positive; therefore, the tachyonic pair formation characterizing our process occurs only when the lower threshold, given by the second expression in Eq. (34), is exceeded. Because the Majorana field has been formulated with full regard to the theory of relativity, even if it is extended to superluminal motions, it is reasonable to assume that the formation process of a Majorana excited state is mediated by a gauge boson, whose four-impulse is given by $4 m_{0}^{2} c^{4} /(J+1 / 2)^{2}$. The mass of this boson will depend on the quantum number $J$.

Although the formation process of an excited state is formally similar to that of beta decay, the ensemble of obtained particles tends to remain together as a composite system, which is one of the cornerstones of the proposed theory. Thus, an interaction force exists between the time-like and spacelike components of the field, and this force can be related to the fifth-force mentioned in other speculative theories [39]. From an algebraic viewpoint, this force is represented precisely by the transformation of similarity that binds the time-like component with the space-like components.

The Lagrangian field that we formulated can be extended symmetrically to the case of antiparticles. In fact, the Majorana equation can be reformulated under the assumption that the allowed solutions have only negative energies. In this case, to avoid the divergences of the Hamiltonian operator, it is be necessary to accept that the excited antiparticle states are represented by composed systems, where tachyons are replaced with antitachyons.

\section{Conclusion}

The neutrino is a particle candidate for testing the Majorana field theory, at least according to the interpretation considered in this study. The neutrino propagates at a velocity very close to that of light and can reach energy in the order of $\mathrm{PeV}$ [38] under optimal conditions, because the ultrarelativistic particle interacts with the tachyonic field. The neutrino is the only particle for which the mass oscillation phenomenon is known [40-41], and recently the MiniBooNE experiment at the Fermilab detected an anomalous event that could be explained by a new neutrino flavour that is different from the three currently known flavours [42].

Moreover, our model may have cosmological implications and, owing to the speculative assumption needed, it could help in explaining the intimate nature of dark matter and dark energy, even if it may 
currently be too risky to undertake this research direction. In particular, in dense regions of the universe, where matter is confined in extremely small spaces and energy is very high, the particles could be accompanied by their tachyonic partners, which suggest that the actual mass is lower in comparison with the mass in a non-ultrarelativistic regime. The dark matter hypothesis of tachyon origin has already been considered by other studies [43-44]. However, the idea that it can be derived directly from ordinary particles through a new decay mechanism is a topic that can stimulate theoretical research beyond the Standard Model.

\section{References}

1. E. Majorana, Relativistic Theory of Particles with Arbitrary Intrinsic Angular Momentum, Il Nuovo Cimento, 9, 335 (1932). English Translation by C.A. Orzalesi in Technical Report, 792, University of Maryland (1968).

2. J.L. Strecker, Infinitesimal Lorentz Transformations, Am. J. Phys., 35(1), 1112 (1967).

3. A.V. Aminova, Lie Algebras of Infinitesimal Projective Transformations of Lorentz Manifolds, Rus. Math. Surv., 50(1), 69 (1995).

4. G. Gamow, Thirty Years that Shook Physics: the Story of Quantum Theory, Doubleday \& Co. Inc., New York (1966).

5. H. Weyl, The Classical Groups: their Invariants and Representations, Princeton University Press, Princeton (1939).

6. C.D. Anderson, The Positive Electron, Phys. Rev., 43(6), 491 (1933).

7. P.A.M. Dirac, The Quantum Theory of Electron, Proc. R. Soc. A, 117(778), 610 (1928).

8. J. Chadwick Existence of Neutron, Proc. R. Soc., 151(873), 479 (1932).

9. C.N. Yang, Fermi's $\beta$-Decay, Int. J. Mod. Phys. A, 27, 1 (2012).

10. C. Strachan, The Theory of Beta-Decay, Elsevier Ltd., New York (1969).

11. S. Esposito, The Physics of Ettore Majorana, Cambridge University Press, Cambridge (2015).

12. R. Casalbuoni, Majorana and the Infinite Component Wave Equations, Proc. Of Science, 37, 1 (2006).

13. D.M. Fradkin, Comment on a Paper by Majorana Concerning Elementary Particles, Am. J. Phys., 34, 314 (1966).

14. A. Bohm, Y. Ne'eman, A.O. Barut, Dynamical Groups and Spectrum Generating Algebras, World Scientific, Singapore (1988).

15. A.O. Barut, Symmetry Properties in Elementary Particle Physics - Review of Hadron Symmetries: Multiplets, Supermultiplets and Infinite Multiplets, Proc. Conference, C70-08-26, 454 (1992).

16. E. Albers, I.T. Grodsky, R.E. Norton, Diseases of Infinite-Component Majorana Field Theories, Phys. Rev., 159(5), 1222 (1967).

17. E.C.G. Surdashan, N. Mukunda, Quantum Theory of the Infinite-Component Majorana Field and the Relation of Spin-Statistics, Phys. Rev., 1(2), 571 (1970).

18. L. Nanni, Determining a Quantum Theory of the Infinite-Component Majorana Field, J. Part. Phys., 2(1), 33 (2018).

19. J. Schwinger, The Majorana Formula, Transactions, 38(1), 170 (1977).

20. E. Recami, G.D. Maccarone, Revisiting the Superluminal Lorentz Transformations and their Group-Theoretical Properties, Lett. Nuovo Cimento, 34(9), 251 (1982).

21. E. Recami, G.D. Maccarone, The introduction of Superluminal Lorentz Transformations: a Revisitation, Fund. Of Phys., 14(5), 367 (1984).

22. E. Recami, R. Mignani, Interpreting Superluminal Lorentz Transformations: Answer to the Comment by Yaccarini, Lett. Nuovo Cimento, 9(9), 357 (1974).

23. J.M. Hill, B.J. Cox, Einstein's Special Relativity Beyond the Speed of Light, Proc. R. Soc. A, 468, 4174 (2012).

24. G. Szekely, The Existence of Superluminal Particle is Consistent with the Kinematics of Einstein's Special Theory of Relativity, Rep. Math. Phys., 72, 133 (2013).

25. A.F. Antippa, A.E. Everett, Tachyons, Causality and Rotational Invariance, Phys. Rev. D, 8, 2352 (1973).

26. M.E. Arons, E.C.G. Surdashan, Lorentz Invariance, Local Field Theory and faster-than-light Particles, Phys. Rev., 173, 1622 (1968).

27. E. Recami, R. Mignani, Crossing Relation Derived from (Extended) Relativity, Int. J. Th. Phys., 12(5), 299 (1975).

28. D. Kirilova, Neutrinos from the Early Universe and Physics Beyond Standard Models, Open Phys., 13, 22 (2016). 
29. J.R. Ellis, Lectures at 1998 CERN Summer School, St. Andrews, Beyond the Standard Model for Hillwalkers [arxiv:hep-ph/9812235].

30. V. Barger, D. Marfatia, K. Whisnant, Progress in the Physics of Massive Neutrinos, Int. J. Mod. Phys., E12, 569 (2003).

31. J. Hansson, On the Origin of Elementary Particle Masses, Prog. in Phys., 10(2), 71 (2014).

32. L. Mikealian, V. Sinev, Neutrino Oscillations at Reactors: What is Next?, Phys, Atomic Nuclei, 63(6), 1002 (2000).

33. G. Bisiacchi, P. Budini, G. Calucci, Majorana Equations for Composite Systems, Phys. Rev., 172, 1508 (1968).

34. L. Nanni, Quantum Theory of Half-integer Spin Free Particle from the Perspective of the Majorana Equation, arxiv:1603.05965[physics.gen-ph].

35. H. Lemke, Quantum Mechanics of Spin-1/2 Tachyons, Il Nuovo Cimento, 35(2), 181 (1976).

36. R.L. Dawe, K.C. Hines, The Physics of Tachyons, Aust. J. Phys., 45, 591 (1992).

37. J.H. Hubbell, Electron-positron pair Production by Photons: a Historical Overview, Rad. Phys. Chem., 75(6), $614(2005)$.

38. U.D. Jentschura, I. Nandori, Neutrino Pair Cerenkov for Tachyonic Neutrinos, Adv. High. En. Phys., 9850312 (2017).

39. E. Fishbach, C. Talmadge, Six Years of Fifth Force, Nature, 356, 207 (1992).

40. Q.R. Ahmad et al., Direct Evidence for Neutrino Flavour Transformation from Neutral-Current Interactions in the Sudbury Neutrino Observatory, Phys. Rev. Lett., 89(1):011301 (2002).

41. P. Fisher, B. Kayser, K.S. McFarland, Neutrino Mass Oscillation, Ann. Rev. Nuc. Part. Sc., 49, 481 (1999).

42. A.A. Aguilar-Arevalo et al., Significant Excess of Electronlike events in the MiniBooNE Short-baseline Neutrino Experiment, Phys. Rev. Lett., 121, 221801 (2018).

43. P.C.W. Davies, Tachyonic Dark Matter, Int. J. Th. Phys., 43(1), 141 (2004).

44. J.S. Bagla, H.K. Jassal, T. Padmanabhan, Cosmology with Tachyon Field as Dark Energy, Phys. Rev. D, 67, 063504 (2003). 